\title{
Electric But Not Eclectic: Thermal Relic Dark Matter for the XENON1T Excess
}

\author{
Joseph Bramante® and Ningqiang Song $\odot$ \\ The McDonald Institute and Department of Physics, Engineering Physics, and Astronomy, Queen's University, \\ Kingston, Ontario, K7L 2S8, Canada and \\ Perimeter Institute for Theoretical Physics, Waterloo, Ontario, N2L 2Y5, Canada
}

(Received 30 June 2020; revised 13 August 2020; accepted 21 September 2020; published 12 October 2020)

\begin{abstract}
The identity of dark matter is being sought with increasingly sensitive and voluminous underground detectors. Recently the XENON1T Collaboration reported excess electronic recoil events, with most of these having recoil energies around 1-30 keV. We show that a straightforward model of inelastic dark matter produced via early Universe thermal freeze-out annihilation can account for the XENON1T excess. Remarkably, this dark matter model consists of a few simple elements: sub-GeV mass Dirac fermion dark matter coupled to a lighter dark photon kinetically mixed with the standard model photon. A scalar field charged under the dark U(1) gauge symmetry can provide a mass for the dark photon and splits the Dirac fermion component state masses by a few $\mathrm{keV}$, which survive in equal abundance and interact inelastically with electrons and nuclei.
\end{abstract}

DOI: 10.1103/PhysRevLett.125.161805

Introduction.-While ample evidence has been collected demonstrating the gravitational influence dark matter (DM) exerts on galaxies and structure formation in the early Universe, DM's origin, couplings, and mass remain a compelling mystery. If DM is a particle with a mass less than a gram, then the predicted flux of DM at Earth's position implies that DM's interactions could be detected with multi-ton-scale detectors, although this will depend on its coupling to known particles. At present, some of the most incisive searches for DM are being conducted in low-background laboratories deep underground.

Recently the XENON1T Collaboration reported an excess of electron recoil events in a 0.65 ton-yr exposure of cooled xenon, with many events having recoil energies around a few keV. Since this xenon search is the most voluminous and sensitive search ever conducted at $\mathrm{keV}$ recoil energies, it is possible these excess events are attributable to a hitherto undetected background process: the beta decay of tritium has been proposed as one such background [1]. However by the same token it is possible that XENON1T has discovered the interactions of a DM particle. Since the XENON1T result was announced, a number of new physics proposals have been put forth to explain the excess [1-24]. However, thus far it has appeared difficult to explain the excess without invoking special DM or dark sector (DS) properties.

Published by the American Physical Society under the terms of the Creative Commons Attribution 4.0 International license. Further distribution of this work must maintain attribution to the author(s) and the published article's title, journal citation, and DOI. Funded by SCOAP .
Here we will demonstrate that a straightforward model of Dirac fermion dark matter, coupled to the standard model (SM) through a dark photon, can account for the observed DM relic abundance and may have been detected as an excess of electron recoil events at XENON1T. Two key features of this model are an inelastic mass splitting of a few $\mathrm{keV}$ between the Dirac fermion component states and that the DM mass is greater than the dark photon mass, so that annihilation of DM in the early Universe proceeds predominantly through annihilation to dark photons. As we will see, the XENON1T excess can be accounted for by inelastic downscatters depositing a few $\mathrm{keV}$ of energy into electrons at XENON1T.

Inelastic dark photon mediated dark matter.-The kinematics and characteristics of inelastic DM models have been studied extensively [25-46]. Inelastic DM mediated by a dark photon has been examined in, e.g., Refs. [36,43,44]. Hereafter our conventions and treatment will follow Ref. [44] most closely, although there are some key differences, since Ref. [44] primarily focused on DM masses in excess of $100 \mathrm{GeV}$, while here we find some details are different for sub-GeV mass DM that explains the XENON1T excess.

We consider a massive dark photon $V$, Dirac fermion $\psi$, and complex scalar $\phi$, all charged under a $U(1)_{D}$ gauge symmetry. The Lagrangian is

$$
\begin{aligned}
\mathcal{L}= & \mathcal{L}_{\mathrm{SM}}+\left|D_{\mu} \phi\right|^{2}-V(\phi)-\frac{1}{4} V_{\mu \nu} V^{\mu \nu}+\epsilon V_{\mu} \partial_{\nu} F^{\mu \nu} \\
& +\bar{\psi}\left(i D_{\mu} \gamma^{\mu}-m_{\psi}\right) \psi+\left(y_{D} \phi \bar{\psi}^{T} C^{-1} \psi+\text { H.c. }\right)
\end{aligned}
$$

where $D_{\mu} \equiv \partial_{\mu}+i g_{D} V_{\mu}$ is the gauge covariant derivative with gauge coupling $\alpha_{D} \equiv g_{D}^{2} / 4 \pi, V_{\mu \nu}$, and $F_{\mu \nu}$ are the dark 
and SM field strength tensors, $C$ is the charge conjugation matrix for $\psi$, and $y_{D}$ is the Yukawa coupling between $\phi$ and $\psi$. $V$ can obtain a mass term of the form $M_{V}^{2} V_{\mu} V^{\mu}$ either through the Stueckelberg mechanism or through coupling to $\phi$. We assume that $\phi$ obtains a vacuum expectation value (vev) $v_{\phi}$ through the machinations of its potential $V(\phi)$. Then the Dirac fermion component mass states, which we label $\chi_{2,1}$ will be split by a mass difference

$\delta \equiv M_{\chi_{2}}-M_{\chi_{1}} \simeq y_{D} v_{\phi}=\operatorname{keV}\left(\frac{y_{d}}{10^{-7}}\right)\left(\frac{v_{\phi}}{10 \mathrm{GeV}}\right)$,

where here we have normalized $\delta \sim \mathrm{keV}$ which will match the XENON1T excess, and the scalar vev to a value which would permit $v_{\phi}$ to generate a sub-GeV mass for $V$, in a DM thermal freeze-out model.

Cosmological production.-In the early Universe the dark sector will be in thermal equilibrium with the SM plasma. Freeze-out of $\chi_{1}$ and $\chi_{2}$ takes place when the temperature of the Universe drops below $M_{\chi}$. We are interested in the "secluded" DM scenario where $M_{V}<M_{\chi}$, so that the annihilations of $\chi_{1}$ and $\chi_{2}$ are dominated by the process $\bar{\chi} \chi \rightarrow V V$. This annihilation cross section is

$$
\sigma v=\frac{\pi \alpha_{D}^{2}}{M_{\chi}^{2}} \sqrt{1-\frac{M_{V}^{2}}{M_{\chi}^{2}}} .
$$

To find the DM relic abundance from freeze-out annihilation, we use the standard formula $[47,48]$

$$
\Omega_{x} h^{2}=\frac{10^{9} x_{f}}{\sqrt{g_{*}} M_{\mathrm{Pl}}\langle\sigma v\rangle \mathrm{GeV}} \approx 0.12,
$$

where $\Omega_{x} h^{2}$ is the comoving relic abundance of DM, $g_{*} \sim 10$ is the number of relativistic degrees of freedom at the time sub-GeV mass DM falls out of equilibrium, $x_{f}=M_{\chi} / T_{f} \sim$ 20 is the mass-normalized freeze-out temperature, and $M_{\mathrm{Pl}}$ is the Planck mass. Using the $\bar{\chi} \chi \rightarrow V V$ annihilation cross section in this relic abundance formula, we find the dark gauge coupling that satisfies DM relic abundance requirements,

$$
\alpha_{D} \simeq 4 \times 10^{-5}\left(\frac{M_{\chi}}{\mathrm{GeV}}\right)\left(1-\frac{M_{V}^{2}}{M_{\chi}^{2}}\right)^{-1 / 4} .
$$

The above treatment of $\chi$ 's relic abundance has neglected the possible effect of $\chi_{1,2}$ mass splitting $\delta$ on thermal freeze-out. This is warranted, since $\delta \ll T_{f}$, and so the mass splitting should not affect freeze-out.

After freeze-out, the interconversion process $\chi_{2} \chi_{2} \leftrightarrow$ $\chi_{1} \chi_{1}$ will be efficient until the temperature of the dark sector drops below some temperature $T_{\mathrm{co}}$. If $T_{\mathrm{co}}<\delta$, the ratio of the number density of $\chi_{2}$ and $\chi_{1}$ is exponentially suppressed $n_{2} / n_{1} \sim e^{-\left(\delta / T_{\mathrm{co}}\right)}$, where the interconversion ceases at

$$
\frac{n_{2}\left\langle\sigma_{\chi_{2} \chi_{2} \rightarrow \chi_{1} \chi_{1}} v\right\rangle}{H} \sim 1
$$

However, in our model we note that the temperature of the dark sector drops rapidly after decoupling from electrons in the thermal bath at $T_{d e}$, since after this $T^{2} \sim T_{D} T_{d e}$, where $T_{D}$ is the DM temperature. In fact, we find that interconversion shuts off at $T_{d e}>T_{\text {co }} \gg \mathrm{keV}$, and so $n_{2} / n_{1} \sim 1$. We estimate $T_{\text {co }}$ as follows: after freeze-out $\chi$ will be nonrelativistic and the interconversion cross section $\sigma_{\chi_{2} \chi_{2} \rightarrow \chi_{1} \chi_{1}} \sim \alpha_{D}^{2} M_{\chi}^{2} / M_{V}^{4}$. The $\chi_{2}$ number density is $n_{2} \sim$ $\left(T^{3} \mathrm{eV} / M_{\chi}\right)$ for a matter-radiation equality temperature $T \sim \mathrm{eV}$, and the DM velocity $v \sim \sqrt{T_{D} / M_{\chi}}$. The conversion rate is compared to Hubble $H \sim T^{2} / M_{\mathrm{Pl}}$. From Eq. (6) it follows that

$$
T_{\mathrm{co}} \sim \frac{M_{V}^{4}}{\alpha_{D}^{2} M_{\chi}^{1 / 2} T_{d e}^{1 / 2} \mathrm{eV} M_{\mathrm{Pl}}} .
$$

For a DM mass $M_{\chi}=1 \mathrm{GeV}$, a mediator mass $M_{V}=0.1 \mathrm{GeV}, \alpha_{D}=4 \times 10^{-5}$, and using an electron kinetic decoupling $T_{d e} \sim \mathrm{MeV}$ (found using similar Hubble rate matching arguments), we find $T_{\mathrm{co}} \sim$ $100 \mathrm{keV} \gg \delta \sim \mathrm{keV}$. This estimate only represents a lower limit on $T_{\mathrm{co}}$. In most of our parameter space, interconversion will cease at temperatures above $100 \mathrm{keV}$. The same estimate can be applied to other DM and mediator masses, and we find $T_{\text {co }}>\delta$ for DM models explaining the XENON1T excess. Consequently we take $n_{2}=n_{1}$ in our analysis.

After freeze-out $\chi_{2}$ may decay to $\chi_{1}$ and SM particles. Since $\delta<2 m_{e}, \chi_{2}$ may only decay to neutrinos and photons. In the presence of V-Z mixing, the $\chi_{2} \rightarrow \chi_{1} \bar{\nu} \nu$ decay rate is given by [36]

$$
\Gamma_{\chi_{2} \rightarrow \chi_{1} \bar{\nu} \nu}=\frac{4 \sin ^{2} \theta_{W}^{4}}{315 \pi^{3}} \frac{G_{F}^{2} \delta^{9}}{M_{V}^{4}} \frac{\epsilon^{2} \alpha_{D}}{\alpha} .
$$

We require the lifetime of $\chi_{2}$ to be longer than the age of universe in order for $\chi_{2}$ to be stable, which gives

$$
\epsilon<0.007 \sqrt{\frac{\alpha}{\alpha_{D}}}\left(\frac{\mathrm{MeV}}{\delta}\right)^{9 / 2}\left(\frac{M_{V}}{100 \mathrm{MeV}}\right)^{2} .
$$

We will be particularly interested in a mass splitting $\delta \sim 3 \mathrm{keV}$, where a decay rate suppression factor of $10^{23}$ is expected relative to the normalization given above, and there is no meaningful constraint on $\epsilon$. We conclude that for parameters around $M_{\chi} \sim 1 \mathrm{GeV}, M_{V} \sim 0.1 \mathrm{GeV}$, and $\alpha_{D} \sim 4 \times 10^{-5}, \epsilon$ is not constrained by decay to neutrinos. While $\chi_{2}$ can also decay to $\chi_{1}$ via the emission of three photons $\chi_{2} \rightarrow \chi_{1}+3 \gamma$, the decay rate in this case is even more suppressed: $\Gamma \propto(\delta / \mathrm{MeV})^{13}$ [36]. Therefore, we 
conclude $\chi_{2}$ is stable for the DM, dark photon, and $\delta$ masses we are interested in.

Lastly, we address $V$ decay. For sub-GeV mass DM, there are bounds on $\chi_{1,2}$ annihilation to SM particles from distortion of the cosmic microwave background (CMB) [49]. However in our setup, $\chi_{1,2}$ annihilates overwhelmingly to $V V$, and so CMB bounds do not apply unless $V$ decays mostly to SM particles. Currently the CMB bound [50] requires that the branching fraction of $V$ to SM particles versus DS particles satisfies $\Gamma_{V \rightarrow \mathrm{SM}} \lesssim 10^{-2}\left(M_{\chi} / \mathrm{GeV}\right) \Gamma_{V \rightarrow \mathrm{DS}}$.

In this paper, we give one example that satisfies the $V$ invisible decay requirement, by adding a less massive, but otherwise identical extra dark photon and fermion $\left(V_{E}, \chi_{E}\right)$ to our Lagrangian (1), where $\chi_{E}$ is charged under both groups $U(1)_{D} \times U(1)_{E}$, with couplings $\alpha_{D}$ and $\alpha_{E}$. In this model, $\chi_{E}$ serves as the invisible decay product of $V$, while $V_{E}$ ensures $\chi_{E}$ freezes out of equilibrium to a negligible relic abundance, through the process $\bar{\chi}_{E} \chi_{E} \rightarrow V_{E} V_{E}$, after which $V_{E}$ decays to SM particles through a photon kinetic mixing of size $\epsilon_{E}$. Crucially, the primary DM field $\chi$ is still only charged under $U(1)_{D}$ with coupling $\alpha_{D}$, so Eq. (5) still fixes $\chi$ 's relic abundance. Then if we require $m_{V}>2 m_{\chi_{E}}>2 m_{V_{E}}, V$ will decay mostly to $\chi_{E}$, and the invisible $V$ decay requirement can be easily satisfied. This can be verified by considering $\left[\left(\Gamma_{V \rightarrow \mathrm{SM}}\right) /\left(\Gamma_{V \rightarrow \mathrm{DS}}\right)\right] \sim$ $\left(M_{V} \alpha \epsilon^{2} / M_{V} \alpha_{D}\right) \sim 10^{-4}\left(\epsilon^{2} / 10^{-6}\right)\left[\left(4 \times 10^{-5}\right) / \alpha_{D}\right]$. Finally, we note that so long as $\left(V_{E}, \chi_{E}\right)$ have masses greater than $10 \mathrm{MeV}$, constraints on $\Delta N_{\text {eff }}$ at recombination and big bang nucleosynthesis can be satisfied [51-53], although CMB stage IV searches may become sensitive to $V_{E}$ as light as $17 \mathrm{MeV}$ [54]. As a benchmark point, we consider $M_{\chi_{E}}=25 \mathrm{MeV}, \quad \alpha_{E}=0.01, \quad M_{V_{E}}=20 \mathrm{MeV}, \quad$ and $\epsilon_{E}=10^{-4}$, which satisfies these $\Delta N_{\text {eff }}$ bounds. The benchmark also yields a small freeze-out $\chi_{E}$ abundance $\Omega_{\chi_{E}} / \Omega_{x} \sim 10^{-8}$, cf. Eq. (5), which avoids the CMB-era $\left(t_{r e} \sim 1 \mathrm{Myr}, z \sim 600\right)$ annihilation bound [50], which is weakened by a factor $\left(\Omega_{\chi_{E}} / \Omega_{x}\right)^{2}$. Similarly, the $\chi_{E}$ produced from later CMB-era $\chi \chi \rightarrow \chi_{E} \chi_{E}$ annihilations are too underabundant to be constrained by Planck. An "onthe-spot" [49] $\chi_{E}$ number density computation $n_{\chi_{E}} \sim$ $2 n_{\chi}^{2}\left\langle\sigma_{\chi \chi} v\right\rangle(1+z)^{3} t_{r e}$ indicates a tiny abundance from $\chi$ annihilation, $n_{\chi_{E}}^{\mathrm{CMB}} / n_{\chi}^{\mathrm{CMB}} \sim 10^{-9}\left(\mathrm{GeV} / \mathrm{M}_{\chi}\right)$.

Downscattering from dark photon dark matter.-In the presence of a heavier DM state $\chi_{2}$ and a lighter state $\chi_{1}$, three possible DM-electron scattering processes may take place in a DM detector: (a) elastic, $\chi_{1(2)}+e \rightarrow \chi_{1(2)}+e$; (b) endothermic, $\chi_{1}+e \rightarrow \chi_{2}+e$; (c) exothermic, $\chi_{2}+e \rightarrow \chi_{1}+e$. The typical recoil energy in process (a) and (b) is $\mu_{\chi e} v^{2} \sim \mathrm{eV}$, which is much smaller than the recoil energy required to explain the XENON1T excess. For the DM model we consider here, (c) exothermic electron scattering would be the only process detected at XENON1T. In this scenario, the electron recoil energy is mainly extracted from the down scattering of $\chi_{2}$.
From energy conservation we can solve for the momentum transfer $q=|\vec{q}|$

$$
q=k \cos \beta \pm \sqrt{k^{2} \cos ^{2} \beta+2 M_{\chi}\left(\delta-E_{R}\right)},
$$

where $\cos \beta$ accounts for the scattering angle between the momentum $\vec{k}$ of $\chi_{2}$ and $\vec{q}$. The maximum and minimum momentum transfer are

$$
q_{\text {min,max }}=\left|k \mp \sqrt{k^{2}+2 M_{\chi}\left(\delta-E_{R}\right)}\right|,
$$

and the minimum velocity

$$
v_{\min }^{2}=\max \left\{\frac{2}{M_{\chi}}\left(E_{R}-\delta\right), 0\right\} .
$$

Following Refs. [55,56] the velocity-averaged differential cross section in exothermic DM scattering reads

$\frac{d\langle\sigma v\rangle}{d E_{R}}=\int_{v_{\min }}^{v_{\max }} \frac{d v}{v} f(v) \int_{q_{\min }}^{q_{\max }} \frac{\sigma_{e}}{2 m_{e}} a_{0}^{2} q d q K\left(E_{R}, q\right)$,

where $a_{0}=1 /\left(m_{e} \alpha\right)$ denotes the Bohr radius with the fine structure constant $\alpha \simeq 1 / 137$, and $K\left(E_{R}, q\right)$ is the atomic ionization factor outlined in Refs. [55,56]. For $E_{R} \sim 2 \mathrm{keV}$, the characteristic momentum transfer $q$ is about tens of $\mathrm{keV}$, which corresponds to $K \sim 0.1$. We take a standard Boltzmann DM velocity distribution $f(v)$, where the angular part has been integrated over. We assume the Earth velocity $v_{e}=240 \mathrm{~km} / \mathrm{s}$ and the escape velocity $v_{\text {esc }}=600 \mathrm{~km} / \mathrm{s}$. The maximum velocity of DM is then $v_{\max }=v_{e}+v_{\text {esc }}$. In the limit where dark photon mass $M_{V}$ is much larger than the momentum transfer, the scattering cross section takes the form $[29,36]$

$$
\sigma_{e}=\frac{16 \pi \epsilon^{2} \alpha \alpha_{D} \mu_{\chi e}^{2}}{M_{V}^{4}}
$$

The electron recoil energy will be smeared by the detector resolution, which to a good approximation can be modeled by [57]

$$
\frac{\sigma_{\mathrm{det}}}{E_{R}}=\frac{a}{\sqrt{E_{R} / \mathrm{keV}}}+b,
$$

where $a=0.3171 \pm 0.0065$ and $b=0.0015 \pm 0.0002$. This gives a resolution of $23 \%$ at $2 \mathrm{keV}$. We take the Gaussian resolution function

$$
\operatorname{Res}\left(E, E_{R}\right)=\frac{1}{\sqrt{2 \pi \sigma_{\mathrm{det}}^{2}}} e^{-\frac{\left(E-E_{R}\right)^{2}}{\sigma_{\mathrm{det}}}} \alpha(E),
$$

which incorporates the efficiency $\alpha\left(E_{R}\right)$ reported in Ref. [1] and can be convoluted with the velocity-averaged cross 
section in Eq. (13) to produce the DM detection rate in the XENON1T detector

$$
\frac{d R}{d E}=N_{T} \frac{\rho_{\chi_{2}}}{M_{\chi}} \int \frac{d\langle\sigma v\rangle}{d E_{R}} \operatorname{Res}\left(E, E_{R}\right) d E_{R},
$$

where $N_{T} \simeq 4.2 \times 10^{27} /$ ton is the number of xenon atoms in the detector, and $\rho_{\chi_{2}}$ is the energy density of $\chi_{2}$. As detailed in the preceding section, it is safe to assume that half the DM particles are in the excited $\chi_{2}$ state for the model parameters we are interested in, in which case $\rho_{\chi_{2}} \simeq 0.15 \mathrm{GeV} / \mathrm{cm}^{3}$.

We show the expected event rate from exothermic scattering in Fig. 1 for a best-fit inelastic mass splitting $\delta=2.8 \mathrm{keV}$. Regardless of $M_{\chi}$, the scattering rate exhibits a sharp peak around $\delta$ before detector resolution smearing. The rate drops abruptly as $E_{R}>\delta$ for $M_{\chi}=0.1 \mathrm{GeV}$, due to a relatively large $v_{\min }$ as can be understood from Eq. (12). Therefore the recoil energy peak for $1 \mathrm{GeV}$ DM tends to be more symmetric. However, this difference in the recoil spectra should not be noticeable in practice, since the recoil energy spectra are appreciably smeared by the detector resolution as given by Eq. (15). We see from the upper panel of Fig. 1 the smeared scattering spectrum with background can describe the XENON1T data quite well. We have fit the XENON1T data [1] in the 1-30 keV range by fixing $\rho_{\chi_{2}}=\rho_{\mathrm{DM}} / 2$ and varying $\sigma_{e}$. We assume $3 \%$ Gaussian error on the efficiency $\alpha(E)$ consistent with Ref. [6]. Although small $M_{\chi} \sim 10 \mathrm{MeV}$ prefers slightly larger $\delta$, we fix $\delta=2.8 \mathrm{keV}$ in the analysis.

The $1 \sigma$ best fit exothermic electron scattering cross section is shown in Fig. 1. Because of the detector resolution and kinematic uniformity of exothermic scattering detailed above, the fit does not change appreciably with DM mass: $\Delta \chi^{2}=\chi_{\text {WIMP }+ \text { bkgd }}^{2}-\chi_{\text {bkgd }}^{2}=-9.8 \rightarrow-10.6$ when $M_{\chi}$ increases from $10 \mathrm{MeV} \rightarrow 1 \mathrm{GeV}$.

In Fig. 2 we show parameter space where DM is produced in the correct relic abundance in the early Universe, and which also predicts an excess of events at XENON1T through exothermic DM-electron scattering. Besides scattering with electrons, dark photon mediated DM may also scatter with nuclei, predominantly through scattering with protons. The per-nucleon scattering cross section against a nucleus with nucleon number $A$ and proton number $Z$ is

$$
\sigma_{n}=\frac{16 \pi \epsilon^{2} \alpha \alpha_{D} \mu_{\chi n}^{2}}{M_{V}^{4}}\left(\frac{Z}{A}\right)^{2}
$$

where $\mu_{\chi n}$ is the DM-nucleon reduced mass. For most low mass nuclei, $Z / A=0.5$, including oxygen at CRESST [58], which sets a leading bound on sub-GeV mass DMnucleon elastic scattering, which we have shown in Fig. 2. At present, CRESST's elastic scattering bound provides the most stringent constraint on nuclear scattering for this
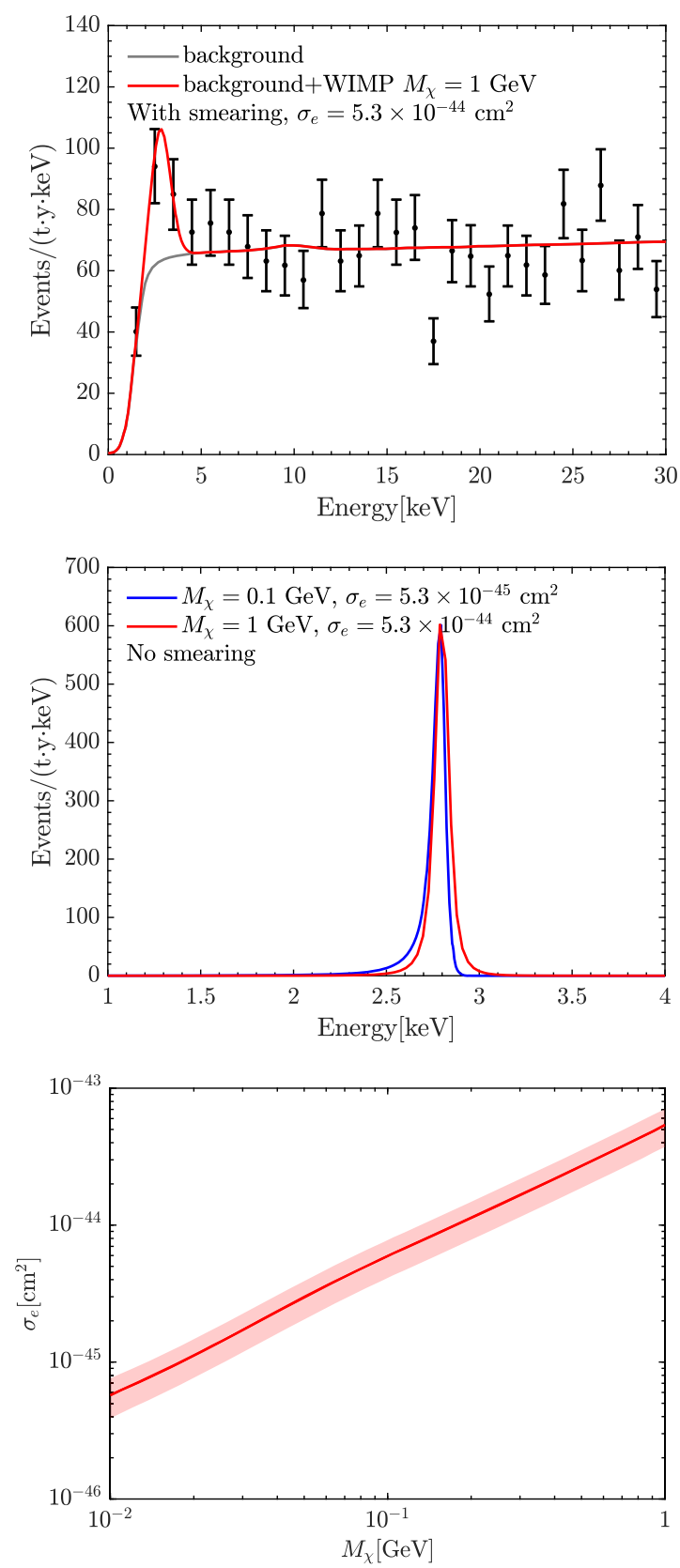

FIG. 1. Exothermic DM-electron scattering fit to the XENON1T excess. Upper: Event rates including both background and exothermic scattering with $M_{\chi}=1 \mathrm{GeV}, \delta=2.8 \mathrm{keV}$ and $\sigma_{e}=$ $4.5 \times 10^{-44} \mathrm{~cm}^{2}$. We assume $\rho_{\chi_{2}}=\rho_{\text {DM }} / 2$ and the detector resolution smearing has been incorporated appropriately. The Xenon1T data and background are extracted from [1]. Middle: Exothermic scattering rates without background and detector smearing. Red and blue lines correspond to $M_{\chi}=1 \mathrm{GeV}$ and $0.1 \mathrm{GeV}$ respectively, for $\sigma_{e}$ indicated. Lower: The best-fit exothermic DM-electron scattering cross-section is given over a range of DM masses, matching the XENON1T electron recoil data. The band shows the $1 \sigma$ preferred region, for $\delta=2.8 \mathrm{keV}$, and $\rho_{\chi_{2}}=\rho_{\mathrm{DM}} / 2$.

model, since the exothermic $\mu_{\chi n} \delta / M_{N} \sim 0.1 \mathrm{keV}$ recoil energy contribution is comparable to elastic recoil energies for sub-GeV DM at CRESST. An exothermic reanalysis of 

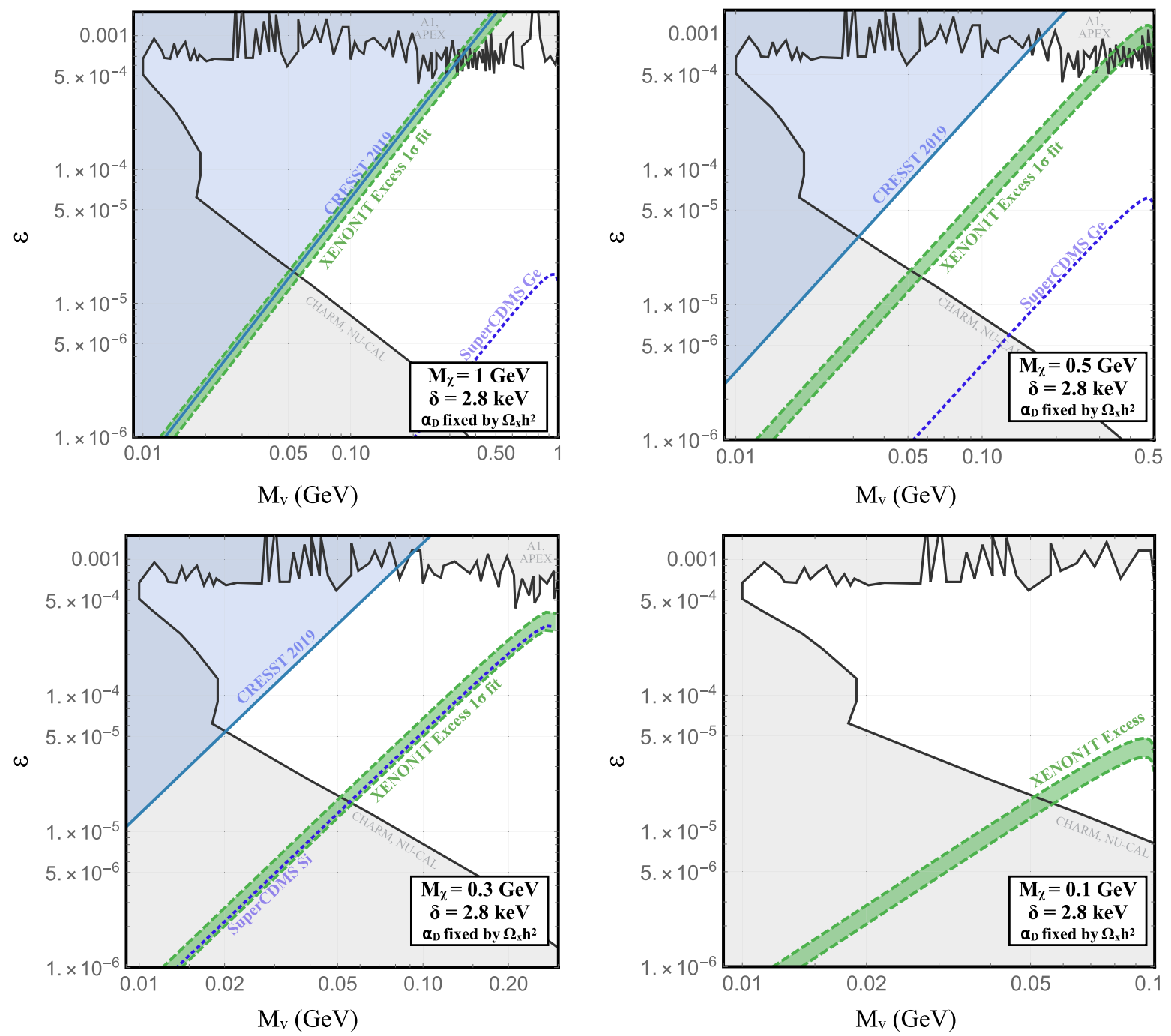

FIG. 2. This figure shows Dirac fermion DM parameters that provide for the observed DM abundance through thermal freeze-out processes in the early Universe, while simultaneously accounting for the observed excess of electron recoil events at XENON1T. Throughout, $\alpha_{D}$ has been fixed to yield the observed cosmological abundance of DM, according to Eq. (5). The green region enclosed by dashed lines shows the $1 \sigma$ best fit inelastic downscattering rate matching the observed XENON1T excess. The electron scattering crosssections corresponding to these parameters are shown in Figure 1. The mass splitting between Dirac fermion component states $\delta$ and DM mass $M_{\chi}$ are indicated. Constraints on dark photons are shown [60-65] (gray) alongside CRESST DM-nucleon scattering bounds [58] (blue), and SuperCDMS Ge/Si projections [66] (dotted).

CRESST recoil data might provide a slightly tighter bound. This would require properly modeling CRESST's low energy recoil backgrounds. For $M_{\chi}=0.1 \mathrm{GeV}$, a weaker bound on DM-nucleon scattering can be derived using the Migdal effect and results from the XENON1T experiment [59]. However, this constraint on $\sigma_{n}$ is too weak to appear in Fig. 2.

Discussion.-We have studied a specific model of inelastic dark photon mediated dark matter, and found that a sub-GeV Dirac fermion coupled to a lighter sub-GeV mass dark photon could account for the XENON1T excess, while simultaneously predicting the correct relic abundance of dark matter through freeze-out annihilation in the early Universe. A crucial feature of this model is a few $\mathrm{keV}$ mass splitting between the component Dirac states, resulting in exothermic electron scattering events at XENON1T.

There are many avenues for future research. While at present, the dark matter-nucleon cross section predicted by this model is too weak to be found out at experiments like CRESST, SuperCDMS, and NEWS-G [58,66,67], these experiments are projected to reach sensitivities that should test this model for Dirac fermion masses down to $0.3 \mathrm{GeV}$, as shown in Fig. 2. In addition, as more electron recoil events are collected and detector resolution improves at xenon experiments like XENON, PandaX, and LZ [68-70], it should become clear whether the electron recoil spectrum exhibits the sharp peak at a few $\mathrm{keV}$ as predicted for exothermic dark photon dark matter in Fig. 1. We look 
forward to pursuing these strategies on the path to unveiling the identity of dark matter.

We thank Fei Gao, Aaron Vincent, and Luc Darme for useful discussions and correspondence. The work of J. B., N. S. is supported by the Natural Sciences and Engineering Research Council of Canada (NSERC). Research at Perimeter Institute is supported in part by the Government of Canada through the Department of Innovation, Science and Economic Development Canada and by the Province of Ontario through the Ministry of Colleges and Universities.

[1] E. Aprile et al. (XENON Collaboration), Observation of excess electronic recoil events in XENON1T, arXiv:2006.09721 [Phys. Rev. D (to be published)].

[2] Juri Smirnov and John F. Beacom, New Freezeout Mechanism for Strongly Interacting Dark Matter, Phys. Rev. Lett. 125, 131301 (2020).

[3] Fuminobu Takahashi, Masaki Yamada, and Wen Yin, this issue, XENON1T Excess from Anomaly-Free Axionlike Dark Matter and Its Implications for Stellar Cooling Anomaly, Phys. Rev. Lett. 125, 161801 (2020).

[4] Kristjan Kannike, Martti Raidal, Hardi Veerme, Alessandro Strumia, and Daniele Teresi, Dark Matter and the XENON1T Electron Recoil Excess, arXiv:2006.10735.

[5] Bartosz Fornal, Pearl Sandick, Jing Shu, Meng Su, and Yue Zhao, preceding Letter, Boosted Dark Matter Interpretation of the XENON1T Excess, Phys. Rev. Lett. 125, 161804 (2020).

[6] Celine Boehm, David G. Cerdeno, Malcolm Fairbairn, Pedro A. N. Machado, and Aaron C. Vincent, Light new physics in XENON1T, arXiv:2006.11250.

[7] G. Alonso-lvarez, F. Ertas, J. Jaeckel, F. Kahlhoefer, and L. J. Thormaehlen, Hidden photon dark matter in the light of XENON1T and stellar cooling, arXiv:2006.11243.

[8] Jatan Buch, Manuel A. Buen-Abad, JiJi Fan, and John Shing Chau Leung, Galactic origin of relativistic bosons and XENON1T excess, arXiv:2006.12488.

[9] Luca Di Luzio, Marco Fedele, Maurizio Giannotti, Federico Mescia, and Enrico Nardi, Solar Axions Cannot Explain the XENON1T Excess, Phys. Rev. Lett. 125, 131804 (2020).

[10] Gil Paz, Alexey A. Petrov, Michele Tammaro, and Jure Zupan, Shining dark matter in Xenon1T, arXiv:2006.12462.

[11] Nicole F. Bell, James B. Dent, Bhaskar Dutta, Sumit Ghosh, Jason Kumar, and Jayden L. Newstead, this issue, Explaining the XENON1T Excess with Luminous Dark Matter, Phys. Rev. Lett. 125, 161803 (2020).

[12] D. Aristizabal Sierra, V. De Romeri, L. J. Flores, and D. K. Papoulias, Light vector mediators facing XENON1T data, Phys. Lett. B 809, 135681 (2020).

[13] Yifan Chen, Jing Shu, Xiao Xue, Guanwen Yuan, and Qiang Yuan, Sun heated MeV-scale dark matter and the XENON1T electron recoil excess, arXiv:2006.12447.

[14] Gongjun Choi, Motoo Suzuki, and Tsutomu T. Yanagida, XENON1T anomaly and its implication for decaying warm dark matter, arXiv:2006.12348.
[15] Keisuke Harigaya, Yuichiro Nakai, and Motoo Suzuki, Inelastic dark matter electron scattering and the XENON1T excess, Phys. Lett. B 809, 135729 (2020).

[16] Hyun Min Lee, Exothermic dark matter for XENON1T excess, arXiv:2006.13183.

[17] R. Primulando, J. Julio, and P. Uttayarat, Collider constraints on a dark matter interpretation of the XENON1T excess, arXiv:2006.13161.

[18] Kazunori Nakayama and Yong Tang, Gravitational production of hidden photon dark matter in light of the XENON1T excess, arXiv:2006.13159.

[19] Amir N. Khan, Can nonstandard neutrino interactions explain the XENON1T spectral excess?, arXiv:2006.12887.

[20] Ujjal Kumar Dey, Tarak Nath Maity, and Tirtha Sankar Ray, Prospects of Migdal effect in the explanation of XENON1T electron recoil excess, arXiv:2006.12529.

[21] Liangliang Su, Wenyu Wang, Lei Wu, Jin Min Yang, and Bin Zhu, Xenon1T anomaly: Inelastic cosmic ray boosted dark matter, arXiv:2006.11837.

[22] Mingxuan Du, Jinhan Liang, Zuowei Liu, Van Que Tran, and Yilun Xue, On-shell mediator dark matter models and the Xenon1T anomaly, arXiv:2006.11949.

[23] Andreas Bally, Sudip Jana, and Andreas Trautner, this issue, Neutrino Self-Interactions and XENON1T Electron Recoil Excess, Phys. Rev. Lett. 125, 161802 (2020).

[24] Qing-Hong Cao, Ran Ding, and Qian-Fei Xiang, Exploring for sub-MeV boosted dark matter from xenon electron direct detection, arXiv:2006.12767.

[25] Lawrence J. Hall, Takeo Moroi, and Hitoshi Murayama, Sneutrino cold dark matter with lepton number violation, Phys. Lett. B 424, 305 (1998).

[26] David Tucker-Smith and Neal Weiner, Inelastic dark matter, Phys. Rev. D 64, 043502 (2001).

[27] David Tucker-Smith and Neal Weiner, The status of inelastic dark matter, Phys. Rev. D 72, 063509 (2005).

[28] Douglas P. Finkbeiner and Neal Weiner, Exciting dark matter and the INTEGRAL/SPI $511 \mathrm{keV}$ signal, Phys. Rev. D 76, 083519 (2007).

[29] Maxim Pospelov, Adam Ritz, and Mikhail B. Voloshin, Secluded WIMP dark matter, Phys. Lett. B 662, 53 (2008).

[30] Spencer Chang, Graham D. Kribs, David Tucker-Smith, and Neal Weiner, Inelastic dark matter in light of DAMA/ LIBRA, Phys. Rev. D 79, 043513 (2009).

[31] Nima Arkani-Hamed, Douglas P. Finkbeiner, Tracy R. Slatyer, and Neal Weiner, A theory of dark matter, Phys. Rev. D 79, 015014 (2009).

[32] Daniele S. M. Alves, Siavosh R. Behbahani, Philip Schuster, and Jay G. Wacker, Composite inelastic dark matter, Phys. Lett. B 692, 323 (2010).

[33] Mariangela Lisanti and Jay G. Wacker, Parity violation in composite inelastic dark matter models, Phys. Rev. D 82, 055023 (2010).

[34] Clifford Cheung, Joshua T. Ruderman, Lian-Tao Wang, and Itay Yavin, Kinetic mixing as the origin of light dark scales, Phys. Rev. D 80, 035008 (2009).

[35] Yanou Cui, David E. Morrissey, David Poland, and Lisa Randall, Candidates for inelastic dark matter, J. High Energy Phys. 05 (2009) 076. 
[36] Brian Batell, Maxim Pospelov, and Adam Ritz, Direct detection of multi-component secluded WIMPs, Phys. Rev. D 79, 115019 (2009).

[37] Patrick J. Fox, Graham D. Kribs, and Tim M. P. Tait, Interpreting dark matter direct detection independently of the local velocity and density distribution, Phys. Rev. D 83, 034007 (2011).

[38] Peter W. Graham, Roni Harnik, Surjeet Rajendran, and Prashant Saraswat, Exothermic dark matter, Phys. Rev. D 82, 063512 (2010).

[39] Rouven Essig, Jared Kaplan, Philip Schuster, and Natalia Toro, On the origin of light dark matter Species, arXiv:1004.0691.

[40] Maxim Pospelov, Neal Weiner, and Itay Yavin, Dark matter detection in two easy steps, Phys. Rev. D 89, 055008 (2014).

[41] Keith R. Dienes, Jason Kumar, Brooks Thomas, and David Yaylali, Dark-Matter Decay as a Complementary Probe of Multicomponent Dark Sectors, Phys. Rev. Lett. 114, 051301 (2015).

[42] G. Barello, Spencer Chang, and Christopher A. Newby, A model independent approach to inelastic dark matter scattering, Phys. Rev. D 90, 094027 (2014).

[43] Eder Izaguirre, Gordan Krnjaic, Philip Schuster, and Natalia Toro, Analyzing the Discovery Potential for Light Dark Matter, Phys. Rev. Lett. 115, 251301 (2015).

[44] Joseph Bramante, Patrick J. Fox, Graham D. Kribs, and Adam Martin, Inelastic frontier: Discovering dark matter at high recoil energy, Phys. Rev. D 94, 115026 (2016).

[45] Luc Darm, Soumya Rao, and Leszek Roszkowski, Light dark Higgs boson in minimal sub-GeV dark matter scenarios, J. High Energy Phys. 03 (2018) 084.

[46] Luc Darm, Soumya Rao, and Leszek Roszkowski, Signatures of dark Higgs boson in light fermionic dark matter scenarios, J. High Energy Phys. 12 (2018) 014.

[47] Joseph Bramante and James Unwin, Superheavy thermal dark matter and primordial asymmetries, J. High Energy Phys. 02 (2017) 119.

[48] Edward W. Kolb and Michael S. Turner, The early Universe, Front. Phys. 69, 1 (1990).

[49] Tracy R. Slatyer, Nikhil Padmanabhan, and Douglas P. Finkbeiner, CMB constraints on WIMP annihilation: Energy absorption during the recombination epoch, Phys. Rev. D 80, 043526 (2009).

[50] N. Aghanim et al. (Planck Collaboration), Planck 2018 results. VI. Cosmological parameters, Astron. Astrophys. 641, A6 (2020).

[51] Kenneth M. Nollett and Gary Steigman, BBN and the CMB constrain light, electromagnetically coupled WIMPs, Phys. Rev. D 89, 083508 (2014).

[52] Cline Boehm, Matthew J. Dolan, and Christopher McCabe, A lower bound on the mass of cold thermal dark matter from Planck, J. Cosmol. Astropart. Phys. 08 (2013) 041.

[53] Anthony Fradette, Maxim Pospelov, Josef Pradler, and Adam Ritz, Cosmological constraints on very dark photons, Phys. Rev. D 90, 035022 (2014).

[54] Masahiro Ibe, Shin Kobayashi, Yuhei Nakayama, and Satoshi Shirai, Cosmological constraint on dark photon from $\mathrm{N}_{\mathrm{eff}}$, J. High Energy Phys. 04 (2020) 009.
[55] B. M. Roberts, V. A. Dzuba, V. V. Flambaum, M. Pospelov, and Y. V. Stadnik, Dark matter scattering on electrons: Accurate calculations of atomic excitations and implications for the DAMA signal, Phys. Rev. D 93, 115037 (2016).

[56] B. M. Roberts and V. V. Flambaum, Electron-interacting dark matter: Implications from DAMA/LIBRA-phase 2 and prospects for liquid xenon detectors and NaI detectors, Phys. Rev. D 100, 063017 (2019).

[57] E. Aprile et al. (XENON Collaboration), Energy resolution and linearity in the $\mathrm{keV}$ to $\mathrm{MeV}$ range measured in XENON1T, Eur. Phys. J. C 80, 785 (2020).

[58] A. H. Abdelhameed et al. (CRESST Collaboration), First results from the CRESST-III low-mass dark matter program, Phys. Rev. D 100, 102002 (2019).

[59] Rouven Essig, Josef Pradler, Mukul Sholapurkar, and Tien-Tien Yu, Relation between the Migdal Effect and Dark Matter-Electron Scattering in Isolated Atoms and Semiconductors, Phys. Rev. Lett. 124, 021801 (2020).

[60] S. Abrahamyan et al. (APEX Collaboration), Search for a New Gauge Boson in Electron-Nucleus Fixed-Target Scattering by the APEX Experiment, Phys. Rev. Lett. 107, 191804 (2011).

[61] Rouven Essig et al., Working group report: New light weakly coupled particles, in Community Summer Study 2013: Snowmass on the Mississippi (CSS2013) Minneapolis, MN, USA, 2013 (Snowmass, Colorado, 2013).

[62] Evgueni Goudzovski (NA48/2), Search for the dark photon in $\pi^{0}$ decays by the NA48/2 experiment at CERN, EPJ Web Conf. 96, 01017 (2015).

[63] H. Merkel, P. Achenbach, C. AyerbeGayoso, T. Beranek, J. Bericic et al., Search at the Mainz Microtron for Light Massive Gauge Bosons Relevant for the Muon g-2 Anomaly, Phys. Rev. Lett. 112, 221802 (2014).

[64] Brian Batell, Rouven Essig, and Ze'ev Surujon, Strong Constraints on Sub-GeV Dark Sectors from SLAC Beam Dump E137, Phys. Rev. Lett. 113, 171802 (2014).

[65] L. Marsicano, M. Battaglieri, M. Bondi, C. D. R. Carvajal, A. Celentano, M. De Napoli, R. De Vita, E. Nardi, M. Raggi, and P. Valente, Novel Way to Search for Light Dark Matter in Lepton Beam-Dump Experiments, Phys. Rev. Lett. 121, 041802 (2018).

[66] R. Agnese et al. (SuperCDMS Collaboration), Projected sensitivity of the SuperCDMS SNOLAB experiment, Phys. Rev. D 95, 082002 (2017).

[67] Q. Arnaud et al. (NEWS-G Collaboration), First results from the NEWS-G direct dark matter search experiment at the LSM, Astropart. Phys. 97, 54 (2018).

[68] E. Aprile et al. (XENON Collaboration), Physics reach of the XENON1T dark matter experiment, J. Cosmol. Astropart. Phys. 04 (2016) 027.

[69] Xiangyi Cui et al. (PandaX-II Collaboration), Dark Matter Results From 54-Ton-Day Exposure of PandaX-II Experiment, Phys. Rev. Lett. 119, 181302 (2017).

[70] B. J. Mount et al., LUX-ZEPLIN (LZ) technical design report, Report No. LBNL-1007256, LBNL, (2017). 